\title{
Influence of mask surface processing on CD-SEM imaging
}

\author{
Marc Hauptmann ${ }^{1}$, Lukas M. Eng ${ }^{2}$, Jan Richter ${ }^{1}$ \\ ${ }^{1}$ Advanced Mask Technology Center GmbH \& Co KG, Rähnitzer Allee 9, 01109 Dresden, Germany \\ ${ }^{2}$ TU Dresden, Institut für Angewandte Photophysik, 01062 Dresden, Germany \\ Phone: +49-351-4048-255 FAX:+ +49-351-4048-9 255 e-mail: Jan.Richter@amtc-dresden.com
}

\begin{abstract}
Photomask processing during mask manufacturing can influence key-parameters of CD-SEM measurement such as measurement accuracy and repeatability. In particular, resist strip and mask clean processes seriously affect the surface properties of CoG-reticles and therefore increase the chrome-CD-measurement uncertainty. In extreme cases, the change of chemo-physical properties of the top Chromium oxide layer can have dramatically impact on the electrons emitted by the surface, utilized by the imaging process of CD SEMs. This change leads to a decreased secondary electron yield, and even more dramatic, induces charging near isolated chromium structures. Although the surface changes appear to be reversible with typical charge decay times in the order of days, the associated charging effect is in severe conflict to the common demand for low cycle times and high measurement accuracy experiments for the CD-measurement processes.
\end{abstract}

In this work, we present fundamental experiments on Chromium oxide layers taking into account the effect of hydrophilicity on the optical as well as on the electrical behavior. We observe that the dependence of secondary electron yield on primary electron energy turns out to be the main issue effecting charging of electrically floating chromium structures. This charging effect thus can be understood in terms of shifting the so-called "iso-electrical point", ultimately resulting in contrast reversion. Furthermore, our data are compared to a simple model respecting electron induced charging during mask surface activation. Our model is supported by numerical calculations of the effective surface potentials near isolated chromium structures. Moreover, the model provides ways to find novel routes for improved surface preparation prior to CD-measurement that allow a good SEM imaging behavior.

\section{Introduction}

With the introduction of Low-Voltage Scanning Electron Microscopy to both wafer and photomask metrology of critical dimensions (CD-SEM), this technique has subsequently evolved in order to supplement optical metrology and to meet the requirements of next-generation semiconductor fabrication. Today's CD-SEMs combine the high spatial resolution and large focus depth of conventional Scanning Electron Microscopy with the demand on high throughput and measurement accuracy associated with volume production. Therefore, a fundamental knowledge concerning the formation of secondary electron (SE) contrast is essential for the development of measurement models

Photomask and Next-Generation Lithography Mask Technology XV,

edited by Toshiyuki Horiuchi, Proc. of SPIE Vol. 7028, 702830, (2008)

0277-786X/08/\$18 - doi: $10.1117 / 12.793106$

Proc. of SPIE Vol. 7028 702830-1 
and algorithms, that the requirements determined by industry roadmaps.

Recently much effort has been made to understand different tool and their behavior. ${ }^{1-4}$ However, modern CD-SEM measurements are now so accurate that effects can be detected that are clearly not only measurement tool related. Thus the artifact of the measurement the mask itself is getting in the focus of a general understanding in metrology. Here, we choose a very pragmatic approach and investigate the occurrence of charging as one of the most critical issue that seriously effects SE contrast. The general physical root cause of charging is that the substrate layer of glass is almost perfectly insulating. Nevertheless it is worthwhile to go into more depths here to understand the interdependence of measurement tool with measurement artifact.

\section{Experimental}

Our starting point is that to achieve best repeatability CD-SEMs working near or shortly below the 2nd isoelectric point (IEP) of chrome that has typical values between 1.5 and $2 \mathrm{kV}$. Working around this point ensures a fast equilibrium of charge on the surface. ${ }^{5,6}$ This has to be understood in the context of charging and charge distribution on isolated conductive layers on insulators such as glass; effects that tend to take an amount of time to stabilize that is in the order of a magnitude of image capturing times. The smaller the net charge on the structure the faster equilibrium is reached and the better the reproducibility. A very evident experiment would then to investigate a process that alters the position of the isoelectrical point. Here, we will investigate surface treatments that may have critical impact on the position of the isoelectric point, which in worst cases is shifted below the working point given by IEP energy, leading to negative charging of floating conductive layers and related effects.

After surface treatment during mask fabrication processes such as clean and resist strip, SEM images of CoG reticles show significant variations of SE contrast, which tend to disturb automated pattern recognition algorithms as well as CD-measurement itself. In our experiments we used a standard binary chrome photomask and investigated the reticle before and after a standard final mask clean process. The most obvious results can be seen on CD-SEM tools with a high acceleration voltage close to $2 \mathrm{kV}$ and high current densities. In extreme cases, signal strength relations of chrome against glass are inverted, as shown in figure 1a. Contrast variations depend on structure design as well. A main characteristic of patterns that show alternated imaging behavior is given by the existence of isolated structures as depicted in figure $1 \mathrm{~b}$. The utilized test design consists of two sub-patterns referred to as negative with isolated features) and positive (without isolated features), containing line-and-space test structures with various line widths. Only floating features show charge induced contrast variation effects, as they are not connected to the surrounding chrome which serves as a ground electrode. The strength of contrast variation effects depends on the electrode size. Hence, dense fields, where a number of chrome lines are connected to a single, plain electrode, show less charging than so-called "Iso-lines", where the electrode

consists of a single line-type chromium layer. Obviously the strongest impact is on isolated dot structures.

Proc. of SPIE Vol. 7028 702830-2 

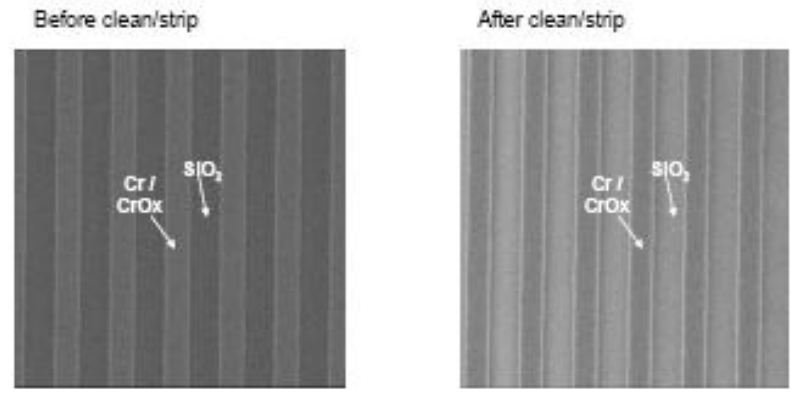

(a) SE contrast variations on CoG reticles (FOV $[3 \mu \mathrm{m}]^{2}, P E$ Acc-Voltage $\left.1.8 \mathrm{kV}\right)$.

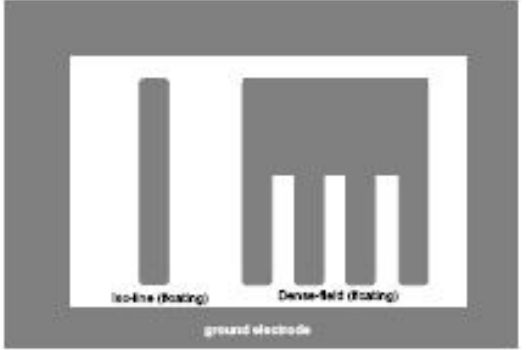

(b) Schematic overview of features showing abnormal SEM contrast after mask surface treatment.

Figure 1: Contrast variation occur on various structure types.

To demonstrate that this effect is by no means an artifact of complicated patterns we modified the clean process to increase the charging. Finally, charging could be demonstrated on standard alignment marks as shown in figure 2. Here the difference between floating and grounded chrome is very clearly visible. Furthermore another byproduct of excess charges is visible too, a charging corona, which leads to an unstable cloudlike image distortion.

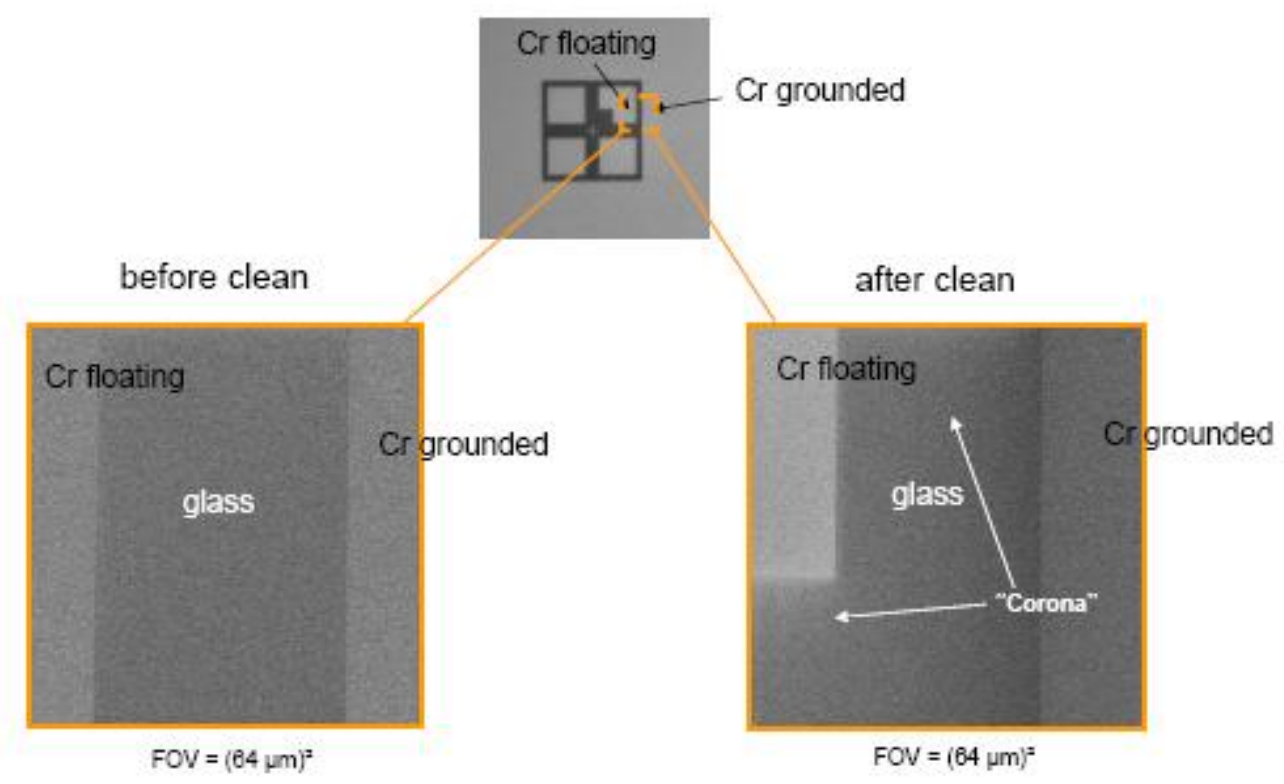

Figure 2: Contrast variations on a CoG reticle at acceleration voltage of $1.8 \mathrm{kV}$. After clean, a signal contrast is observed in low magnification between floating and ground layer floating-to-ground contrast). This is caused by charging of the floating electrode. A charged electrode is usually surrounded by an area of increased SE emission ("charging corona"). 
Although the charging corona is a clear evidence of charge induced differences in the CD-SEM image, a direct experiment confirmed that charging is indeed the root cause of the image contrast changes. The difference of floating to grounded chrome areas allows the following experiment experiment. Here, a formerly floating electrode was contacted to the surrounding ground electrode by means of a focused Ion Beam (FIB). The result is depicted in figure 3. Immediately after contacting the left down electrode the contrast changes dramatically and the formerly floating electrode has now a contrast matching the surrounding ground electrode and is clearly different from the remaining floating electrodes.

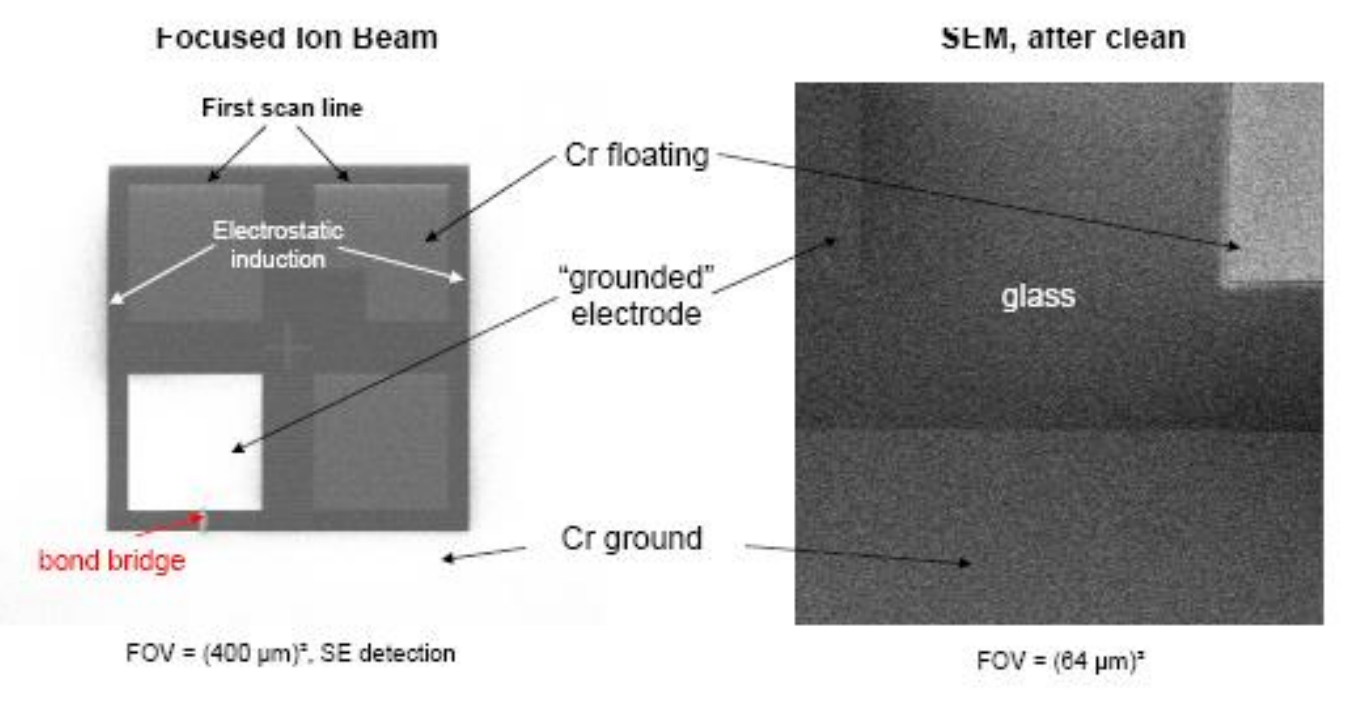

Figure 3: Charging effects tend to adjust immediately within the first scan due to high ion currents and low scanning rates. Electrodes connected to ground potential (bottm left) do not show any charging in contrast to floating layers (with inverted contrast between floating and ground electrode in the SEM).

The next step in understanding the observed behaviour after mask cleaning was to investigate the physical properties of the mask surface. Here, two standard techniques are reported one being Vis-spectroscopy and the other contact angle measurements. During mask cleaning, an enhancement of surface wet ability is achieved, when adsorbed organic components are removed via oxidation e.g. using sulfuracid-hydrogenperoxide solution (SPM, acid environment) or ammoniumhydroxide-hydrogenperoxide solution (alkaline environment). This is accompanied by an increase of surface polarity of chromium oxide which results in the formation of surface OH-groups and enhanced attraction of surface water. ${ }^{7-10}$ Since surface OH-groups again mostly act as reactive sites concerning the adsorption of organic species from the ambient air, a self-passivating behavior is associated with the hydrophilization of the mask surface and reaction times within the order of magnitude of days (this also the needed relaxation time after clean to measure these masks with normal contrast) . Properties, related to the constitution of the chromium oxide surface, such as reflectivity and contact angle, should therefore show equivalent behavior. Finally, a correlation can be found between 
the occurrence of SEM contrast variations, a change of the optical behavior of the top chromium oxide layer and the state of hydrophilicity of the oxide surface. Generally, surface reflectivity is increased after mask cleaning especially in the range from 200 to $600 \mathrm{~nm}$, while the mask is accordingly showing alternated SEM imaging behavior (figure 4). The enhancement is most likely caused by the excitation of surface dipole moments, when the polar surface is irradiated with ultraviolet (and visible) light, and the neglect able adsorption of UV radiation by unpolar, organic adsorbents. The strength of SEM contrast variations varies with the reflectivity of the chromium oxide surface. A relaxation of the SEM imaging behavior accompanying a change of the optical characteristics in direction of the inertial conditions can thereby be observed. Additionally, the corresponding change of hydrophilicity can be visualized using contact angle measurements, showing that a more hydrophobic surface is associated with lowered strength of SEM charging and decreased reflectivity in the near UV. The corresponding spectra show higher site-to-site variation with proceeding passivation of the cleaned ARC-surface, which is accompanied by an enhanced standard deviation of the according contact angle values. A possible interpretation could be that the adsorption of organic molecules from ambient air is depending on the lateral position on the photomask, probably as a consequence of variations in the density of reactive $\mathrm{OH}$-groups acting as adsorption sites, since the mask is rotated with high velocity during cleaning. The likewise resulting anisotropic adsorption of anions at the chromium oxide surface may point to a correlation between the degree of adsorption and the strength of relaxation, since reflectivity increases between 600 and $800 \mathrm{~nm}$ and remains constant above $800 \mathrm{~nm}$ with proceeding surface passivation. A correlation with the amount of surface-adsorbed water can not be significantly varied, because detector performance gets poor when approaching the IR-range.

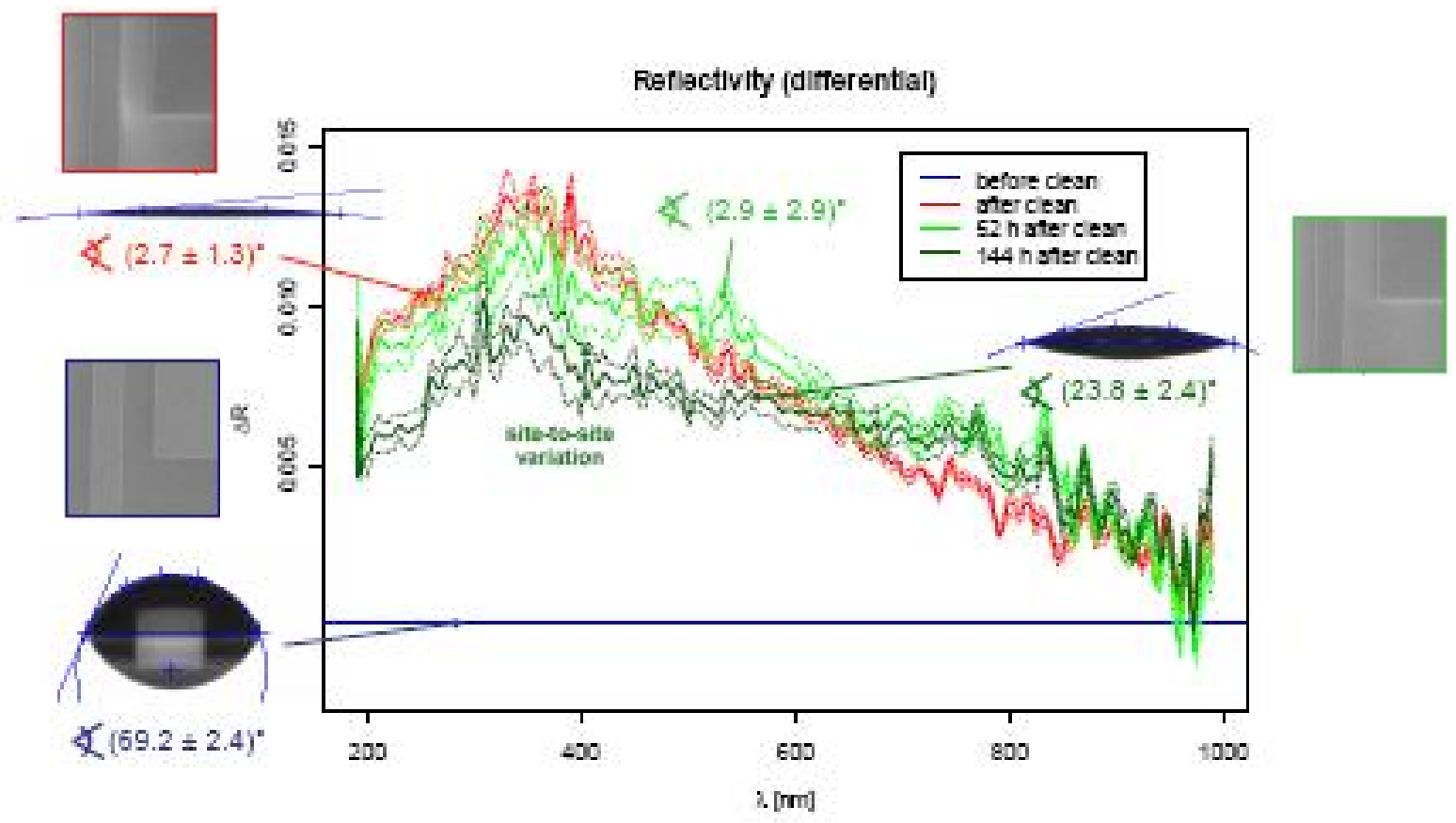


Figure 4: Change of reflectivity with respect of the initial state of the mask surface with reflectance (blue line). The reflectance shows a significant increase in the near UV (200-600 nm) after surface cleaning (red line), which corresponds to a hydrophilic surface state, followed by slow relaxation to a more hydrophobic surface composition (light and dark green line) due to the re-adsorption of olefins removed during clean procedure. While a steady increase of reflectance between 600 and $800 \mathrm{~nm}$ is probably caused by surface diffusion of adsorbed an-and cat ions, reflectivity remains at a constant level above $800 \mathrm{~nm}$, which is most likely due to surface water. The rising site-to-site-deviation of the reflectance (dashed lines) during ongoing surface passivation is associated with a similar behavior of the contact angle.

The chemical and physical properties of photomask anti-reflective coatings are mainly governed by the top chromium oxide layer due to its catalytic and electronic properties. The catalytic behavior can thereby be explained in the picture of ligand-feld stabilization energy. It turns out, that the appearance of SEM charging can be related to a change in the hygroscopic behavior of the oxide surface from more hydrophobic (associated with the adsorption of organic molecules) to hydrophilic (corresponding to the development of surface OH-groups after mask surface cleaning) characteristic. As the re-adsorption of organic molecules from ambient air is mainly driven by the density of surface $\mathrm{OH}$-groups, surface passivation, which occurs within relaxation times in the order of days and is correlated to the re-establishment of undisturbed imaging behavior in the SEM, may be suppressed due to the adsorption of anions (such as sulfates) also changing the electron configuration at the oxide surface. Hydrophility of the chromium oxide surface influences the properties of SE directly, by a slight shift of the materials work function to lower values, and indirectly by an increased density of adsorbed water, which is accompanied by a rise of surface conductivity and increased probability for SE scattering on the water dipole moments. As even under high-vacuum conditions hydrophilic surfaces are covered with a monolayer of water-molecules, their increased surface density is expected to be linked to the shift of the IEP towards higher PE energies and the occurrence of SEM contrast variations.

\section{Conclusion}

In the present work, it was shown, that secondary electron contrast variations on binary photomasks occurring in the low-voltage SEM are related to the electron induced charging of isolated floating conductive layers during electron irradiation. The appearance of charging on conductive layers after mask surface treatment could successfully be referred to a shift of the iso-electric point below the SEM working point. As a consequence, SE electron signal on quartz regions adjacent to floating chrome is disturbed, as the surface equilibrium potential re-establishes and the negative potential above the charged electrode leads to SE detection, acceleration and multiplication in the SEM chamber. The shift of the isoelectrical point is thereby caused by an increased hydrophilicity of the top chromium oxide layer surface, which is achieved during mask cleaning by the removal of organic adsorbents and the formation of surface OH-groups. The natural relaxation of surface hydrophilicity and related SEM imaging behavior is caused by the adsorption of olefins from ambient air and has been measured by 
contact angle and Vis-spectroscopy.

One of the more general findings of this paper is therefore the ongoing interdependence of different production steps in nanotechnology. This interdependence is well accepted for process steps like etch or develop. However, this work suggests that measurement and characterization steps cannot be seen as isolated events anymore, but depend strongly on the history of the sample.

\section{Acknowledgements}

AMTC is a joint venture of AMD, Qimonda and Toppan Photomasks. This work has been partially funded by the German Ministry of Education and Research (BMBF) under Contract No. 01M3154A (Abbildungsmethodiken fuer nanoelektrische Bauelemente).

\section{References}

1. Measurement tool influence on CD results on photolithographic masks, Jan Richter, Roman Liebe, Frank Laske, Jens Rudolf, Torben Heins, Proc. SPIE Vol. 6283 May 2006

2. Systematic investigation of $C D$ metrology tool response to sidewall profile variation on a COG test mask, F. Gans, R. Liebe, T. Heins, J. Richter, W. Häßler-Grohne, C. G. Frase, B. Bodermann, S. Czerkas, K. Dirscherl, H. Bosse, Proc. SPIE Vol. 6281 Jun 2006

3. Matching of different CD-metrology tools for global CD signature on photomasks, E.-M. Zerbe, T. Marschner, J. Richter, C. Utzny, Proc. SPIE Vol. 660714 May 2007

4. Influences on accuracy of SEM based CD mask metrology with a view on the $32 \mathrm{~nm}$ node, $\mathrm{W}$. Häßler-Grohne, C.G. Frase, D. Gnieser, H. Bosse, J. Richter, A. Wiswesser, Submitted to Proc. SPIE 2008

5. The angular distribution of the secondary electrons of Nickel, J.L. Jonker, Philips Res. Rep. (6), pp. 372-387, 1951

6. Charging effects in the SEM, T.J. Shaner, R.D. Van Veld, J. Phys. E (4), pp. 633-637, 1971

7. Mechanism of Anion Adsorption at the Ferric and Chromic Oxide/Water Interface, D.E. Yates, T.W. Healy, J. Colloid Interface Sci (Vol. 52)2, pp. 222-227 (August 1975) 
8. Improving photomask surface properties through a combination of dry and wet cleaning steps, $\mathrm{F}$. Eschbach, D. Tanzil, M. Kovalchick, U. Dietze, M. Liu, F. Xu, Proc. SPIE (5446), pp. 209-217 (2004)

9. Surface $\mathrm{OH}$ group governing adsorption properties of metal oxide films, S. Takeda, M. Fukawa, Y. Hayashi, K. Matsumoto, Thin Solid Films 339, pp. 220-224 (1999)

10. Adsorption and Catalysis on Transition Metals and Their Oxides, V.F. Kiselev, O.V. Krylov, Springer Series in Surface Sciences 9 (Heidelberg, 1989) 